\title{
Research on Segmentation of Vertebral Bodies from Spinal MR Images based on Gauss Weighted and Local Contraction
}

\author{
Sui Dan ${ }^{1,2, *}$, Jiao Zhen ${ }^{1}$ and Yang Xinfeng ${ }^{3}$ \\ ${ }^{1}$ Anyang Normal University, Henan, Anyang, 455000, P.R. China; ${ }^{2}$ Wuhan University of Technology, Hubei, Wuhan, \\ 430070, P.R. China; ${ }^{3}$ Nanyang Institute of Technology, Henan, Nanyang, 473004, P.R. China
}

\begin{abstract}
This paper present a segmentation of vertebral bodies from spinal MR images based on neighborhood information Gauss weighted and local contraction. First use a cut-off window $(5 \times 5)$ around each pixel and stack the gray values inside the window into a vector, adopt the Gaussian kernel function to incorporate local spatial information, an adaptive local scaling parameter is used to refine the segmentation rather than a fixed scaling parameter to avoid the manually tuned parameter. Finally, the built affinity is introduced into the segmentation process by using a graph-based method to achieve the complete target. Extensively experiments show that the present method can segment the vertebral bodies smoothly and clearly, and it has stronger anti-noise property and higher segmentation precision than the conventional methods. The robust and accurate result of segmentation should serve image registration and the analysis of spinal deformities. It is a general method for segmenting object that can develop to segment other tissues and organs.
\end{abstract}

Keywords: Gauss weighted, local contraction, local neighborhood information, MR image, segmentation of vertebral bodies.

\section{INTRODUCTION}

Due to the aging of the society and the increasing of work pressure, a large population has the spondylopathy, such as pleurapophysis, slipped disc and spinal stenosis, which influence lives of people in various trades. Spinal anatomica structure and circum-structure of the vertebrae are very complicated, thus the clinician must has good localization and good knowledge of the vertebrae. The recognition of the vertebral body is the main key of improving efficiency, accuracy and security of the operation in clinical practice [1].

Physicians often make their decisions in diagnosis and treatment of spine with the help of CT and MR images. CT images show reasonably high resolution and give good visualization of the bone, and the vertebral body segmentation is very simple using the thresholding method. MR makes full use of a strong magnetic field (B0 field) and radiofrequency pulses for generating images [2]. If the image intensity is determined by the $\mathrm{T} 1$ relaxation, it is $\mathrm{T} 1$-weighted imaging. If the image intensity is determined by the $\mathrm{T} 1$ relaxation, it is T2-wei^ited imaging. T1-weighted scans work well for differentiating fat from water with water appearing darker and fat brighter. This scans are obtained to observe the anatomical structure of the spine. T2-weighted scans are another basic type. Like the T1-weighted scan, fat is differentiated from water but in this case fat shows darker, and water lighter. In the case of spinal study, the cerebrospinal fluid will be lighter in T2-weighted images [3,4]. This scans are acquired to examine the pathologic change of the spine.
The segmentation of vertebral bodies in MR images is much challenging and complex due to the relatively variations in soft tissue contrast and artifacts like radio-frequency inhomogeneity. There is little work on the vertebral body extraction from the sagittal MR spinal images.

Gamio et al. applied graph-cut to segment MR T1weighted sagittal images of the spine. Graph-cut is an unsupervised method and does not require initialization. Using methods based on graph cut, an image is usually segmented into several distinct regions rather than the target and the background, and the pixels have high similarity within each region. A weighted graph is constructed, where nodes of the graph correspond to image pixels, and the weight of the edge reflects the similarity between two joined nodes. Solving the eigenvectors and eigenvalues of the affinity matrix performs the image segmentation [5-7]. This method defined a partitioning criterion that maximizes the total similarity within groups and minimizes the total similarity between different groups. Gamio et al. segmented the vertebral body using windowed histograms of intensity as the most promising features. Due to the usage of the simple statistical characteristics of local histogram, Gamio algorithm is not a good choice for segmenting the images with same statistical characteristics of local histogram and lowcontrast objects [8].

Because of the influence of noise and anisotropy factor in imaging technology, pixel gray value of the same anatomical organizational structure in image will change greatly; this feature does not accurately describe an image with just a single pixel gray value, so we use the feature of gray level in the neighborhood information to describe an image, which introduces image space structure information. Based on the features we propose a new similarity matrix structure, based 
on the neighborhood information and Gauss weighted ChiSquare distance, and the use of adaptive local contraction method Zelnik put forward to adjust the scale parameter, to achieve accurate segmentation of $2 \mathrm{D}$ vertebral body. Even in the noisy case, the algorithm can fast and accurately segment the vertebral body, for normal and pathological vertebral body, vertebral body outline this paper algorithm segmented were smooth and clear, as a general segmentation method, the algorithm can be extended to other organ segmentation.

\section{BASIC THEORY}

The image segmentation technology based on graph theory is a new image segmentation technology, because of good properties, are widely concerned by researchers. The technology is a kind of combinatorial optimization method; one image can be mapped into a network diagram, structure similarity matrix, define a criterion function to complete the image segmentation [9].

In the segmentation algorithm based on graph theory, how to structure a similarity matrix is a hot research topic [10]. The similarity of between two nodes (pixels) $v_{i}$ and

$v_{j}$ in the image is expressed as:

$W_{i j}=e^{-\frac{d^{2}(i, j)}{\sigma^{2}}}$

Among them, $d(i, j)$ is the distance function for vertex $v_{i}$ and $v_{j}, \sigma$ is a scale factor.

In 2001, Ng et al. proposed NJW algorithm [11], which use the gray information of image pixel structure similarity matrix algorithm, that is:

$W_{i j}=e^{-\frac{\left\|I_{i}-I_{j}\right\|^{2}}{\sigma^{2}}}$

Among them, $I_{i}$ and $I_{j}$ said the gray of the pixel is $v_{i}$ and $v_{j}$ in the image. The NJW algorithm is sensitive to noise and image artifacts.

Gamio et al. [12] proposed a similarity matrix using image local information, expressed as:

$$
\begin{aligned}
& W_{i j}=e^{-\frac{x_{i j}^{2}}{\sigma^{2}}} \\
& x_{i j}^{2}=\frac{1}{2} \sum_{k=1}^{n k} \frac{\left(h_{i}(k)-h_{j}(k)\right)^{2}}{h_{i}(k)+h_{j}(k)}
\end{aligned}
$$

Among them, $h_{i}(k)$ is the $k$ element of local histogram of the $i$ pixel, $n k$ is the number of local histogram elements. This method can improve the segmentation accuracy in a certain extent. Chi-square distance is a measure to effective measure the similarity of histogram. When there is no obvious difference in the statistical characteristics of image, it cannot accurately target segmentation.
Shi et al. [13] proposed an new similarity matrix based on image gray and interference contour, expressed as:

$W_{i j}=\sqrt{W_{i j}^{I} \times W_{i j}^{C}}+\alpha W_{i j}^{C}$
$W_{i j}^{I}=e^{-\left\|X_{i}-X_{j}\right\|^{2} / \sigma_{x}-\left\|I_{i}-I_{j}\right\|^{2} / \sigma_{I}}$
$W_{i j}^{C}=e^{-\max _{x \in \operatorname{line}(i, j)}\|\operatorname{Edge}(x)\|^{2} / \sigma_{C}}$

Among them, $X_{i}$ said the position of pixel $v_{i}$ in image; line $(i, j)$ said a straight line connected pixel $i$ and $j$; $\operatorname{Edge}(x)$ said $x$ edge strength; $\sigma_{x}, \sigma_{I}$ and $\sigma_{C}$ is the scale parameter; $\alpha$ is the weight of $W_{i j}^{C}$. This method can obtain satisfactory effect in the image segmentation, the speed of algorithm is faster, but the parameter setting is more complex.

\section{SEGMENTATION OF VERTEBRAL BODIES FROM SPINAL MR IMAGES BASED ON NEIGH- BORHOOD INFORMATION GAUSS WEIGHTED AND LOCAL CONTRACTION}

In the image segmentation based on graph theory, the most important is to structure similarity matrix, that is, how to measure the distance between two points and select the appropriate scale parameter. This paper used the idea of NJW algorithm in spine MR image segmentation at the first time, and structured a new similarity matrix based on neighborhood information and Gauss weighted chi-square distance in the algorithm, while the introduction of local contraction is high vertebral segmentation accuracy.

In order to segment vertebral body from spine MR image, we propose a new similarity matrix, which overcome the shortcomings above three kind of traditional method extracting target.

\subsection{Structure Similarity Matrix}

\subsubsection{Chi-Square Distance Based on Neighborhood In- formation and Gauss Weighted}

In NJW algorithm, the structure of similarity matrix only considered the single pixel gray value, the same structure pixel gray value changes or the surrounding organs similar grey value leads to wrong clustering and segmentation results. The local neighborhood information of image is an important feature description of an image, introduce the local neighborhood information of the image, that is $h(i)$ :

$h(i)=\sum_{k \in N_{n}} v_{i}(k)$

Wherein, $N_{n}$ said the neighborhood of pixel $v_{i}, v_{i}(k)$ said the $k$ pixel in the neighborhood of pixel $v_{i}$.

Using all pixels values in the neighborhood gray instead single pixel value, using the Gauss function weighted up, 
therefore, the chi-square distance between two nodes (pixels) $v_{i}$ and $v_{k}$ in the image is expressed as:

$d^{2}(i, j)=\frac{1}{2} \sum_{k=1}^{n} w_{k} \frac{\left(v_{i}(k)-v_{j}(k)\right)^{2}}{v_{i}(k)+v_{j}(k)}, \sum_{k=1}^{n} w_{k}=1, w_{k} \geq 0$

In the formula, $v_{i}(k)$ said the gray value of pixel $k$ in the $5 \times 5$ neighborhood of one node, $w k$ is feature weight. Feature weight $w k$ assigned according to the certain prior information, the distance from node is nearer, and the pixel feature weight is larger, on the contrary, the pixel feature weight is smaller. Use Gauss kernel function respectively given feature weights to 25 pixels in neighborhood of the pixel, thus the neighborhood information fusion together, better describes the similarity between two pixels.

\subsubsection{Local Contraction}

When use NJW algorithm to structure similarity matrix, need a scale parameter to control the impact on the similarity matrix of the distance between two sample nodes $v_{i}$ and $v_{j}$, and structure a suitable similarity matrix, improve the clustering performance and the accuracy of segmentation results. The algorithm is based on the clustering effect of mean $\mathrm{K}$ (whether given the most compact clustering) to determine the appropriate scale parameter. Therefore, before finding the right parameters, must repeat eigenvalue iterative and $\mathrm{K}$ means algorithm decomposition, need manually adjust the scale parameter to eventually converge to the optimal solution. Thus, the algorithm has certain limitations, and the amount of calculation is relatively large.

Zelnik et al. proposed a thought called local contraction [14]. Local contraction don't select a scale parameter $\sigma$ for the whole sample set, but select $\sigma_{i}$ for each sample point $v_{i}$, the distance from sample point $v_{i}$ to $v_{j}$ is said $d\left(v_{i}, v_{j}\right) / \sigma_{i}$. Among them, $\sigma_{i}=d\left(v_{i}, v_{k}\right), v_{k}$ is the $K$ close neighbor of ample point $v_{i}$. In the experiment, the general selection is $K=7$, this is a experience value, Taking this value make the clustering effect of the algorithm best.

Therefore, in order to improve the accuracy of clustering and segmentation, we introduce the idea of local contraction, for every pixel adaptively select one $\sigma_{i}$, so as to structure a new similarity matrix $W$. Each element in similarity matrix $W$ is said $W_{i j}=e^{-x^{2}\left(\mathrm{v}_{i}, \mathrm{v}_{j}\right) / \sigma_{i} \sigma_{j}}=e^{-d^{2}\left(\mathrm{v}_{i}, \mathrm{v}_{j}\right) / \sigma_{i} \sigma_{j}}$, Wherein $\sigma_{i}=d\left(v_{i}, v_{k}\right)$.

\subsection{Segmentation}

Based on constructing the new similarity matrix, twodimensional vertebral segmentation algorithm based on optimization criterion of graph normalization is proposed, combined with mathematical morphology realizes twodimensional segmentation and display of vertebral body. In the implementing process of the method, the number of clusters $C=40$ (in other organs segmentation, it can be adjusted accordingly), need manual use the mouse to select the vertebral body to be displayed, one of the parameters don't need adjust, can get accurate segmentation results.

The algorithm proposed in this paper consists 6 steps, as follows:

Step 1: in the 2D spine MR image, manual select the vertebral body need to be displayed;

Step 2: use the method in subclause 3.1 to structure the similarity matrix;

Step 3: calculate the Laplasse matrix proposed in the NJW algorithm;

Step 4: find the largest $C$ eigenvalues in the Laplasse matrix;

Step 5: use K mean to cluster the former $C$ feature vectors, get the clustering result of the image;

Step 6: use mathematical morphology to segment and trim the edge of the vertebral body, get the vertebral body image and vertebral body edge image after image segmentation.

\section{EXPERIMENT}

\subsection{Experimental Data}

The experimental data is from the anyang Central Hospital, equipment is 3.0T magnetic resonance, MR images were collected from 100 pieces of patients, include two modes of 2D sagittal T1 weighted and T2 weighted, the distribution of age range was 40 65 years old. Image acquisition parameters were: resolution is $0.5859 \mathrm{~mm}$, thickness is $4 \mathrm{~mm}$, image size is $512 \times 512$ pixels, the interlayer spacing is $0.4 \mathrm{~mm}$. MR image is divided into 4 categories: the T1 weighted health population spinal images, T1 weighted patients' spinal images, the T2 weighted health population spinal images, T2 weighted patients' spinal images. In order to verify the validity and feasibility of the algorithm in this paper, we do qualitative analysis for the algorithm in this paper the classical NJW algorithm, Gamio algorithm, and Shi algorithm. Clinical expert manual segmentation results as the gold standard, quantitative analysis the accuracy of the algorithm in this paper. As a general segmentation method, this algorithm can also be extended to other organs segmentation, such as extract the brain tumors from enhanced MRT1 weighted image.

The experimental hardware environment: Intel Core Duo (2.53 GHz) processor, $2 \mathrm{~GB}$ ram; Software environment: Windows 7 operating system; software tools: Matlab 2008a and Visual Studio 2008.

\subsection{Experimental Results}

Experiment 1: T1 weighted images, patients with normal vertebral body

Manual segmentation algorithm: Fig. (1) is the vertebral spine experimental results clinical expert manual segmented from MR image; the red solid line is vertebral body contour 
segmented, right figure is the vertebral body extracted. The manual segmentation results as the gold standard to validate the accuracy and effectiveness of the improved segmentation algorithm for the vertebral body.

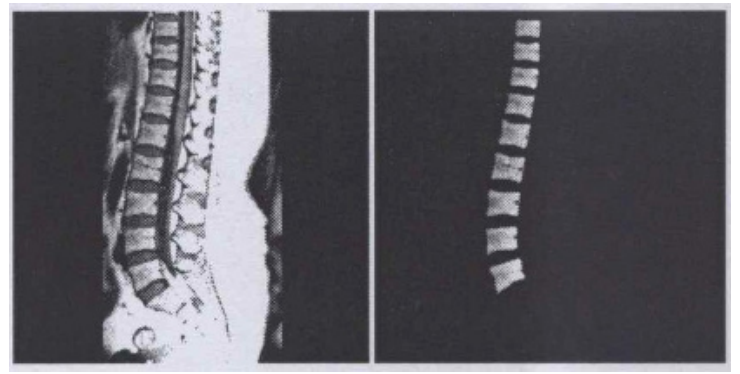

Fig. (1). Gold Standard: the boundaries (red line) and segmentation results of the vertebral bodies.

NJW algorithm: use gray value of each pixel as the feature, and not use local contraction to do vertebral automatic segmentation experiments from the spine MRI, the experimental results is shown in Fig. (2a), the red solid is vertebral body contour segmented. Know from the experimental results, at the binding of vertebral body and the adjacent tissue, gray is close, cause the over segmentation phenomenon (Fig. 2a) blue arrow); while in the other vertebral segmentation, appear less segmentation phenomenon (Fig. 2a) red arrow). Among them, the parameter is set to $\sigma=0.2, C=35$.

Gamio algorithm: use the window histogram as image significant features, without considering the spatial information; at the same time, the scale factor is a constant, need repeat manual adjustment in order to get better segmentation results, Fig. (2b) shows the segmentation results of Gamio algorithm. Among them, the parameter is set to $\sigma=0.45$, $C=35$.

Shi algorithm: use the gray information and the interference contour as classification features of images, does not consider the neighborhood information, the segmentation results are shown in Fig. (2c). Among them, the parameter is set to $\sigma_{x}=\infty, \sigma_{I}=0.12, \sigma_{C}=0.08, \alpha=1, C=65$.

The improved algorithm in this paper: considered the spatial neighborhood information of pixels, 25 gray values of pixels in the $5 \times 5$ pixels neighborhood were weighted using Gauss function, combined with local contraction to complete vertebral segmentation from spinal MR images, the experimental results is shown in Fig. (2d), red solid line is the vertebral contours segmented. Even if the gray of body and surrounding tissue is close, this algorithm can also accurately segment the vertebral body, smooth and clear, overcome the over segmentation and the less segmentation phenomenon in the NJW algorithm, the segmentation of vertebral contours and vertebral body shape is close to the manual segmentation results.

Experiment 2: T1 weighted images, the lesions occur in vertebral body of patients

Patients vertebral are with degeneration, intervertebral is disc herniation. Fig. (3) shows the vertebral body edges a clinical expert manual segmented and the vertebral body extracted. Although the vertebrae degenerative changes, the
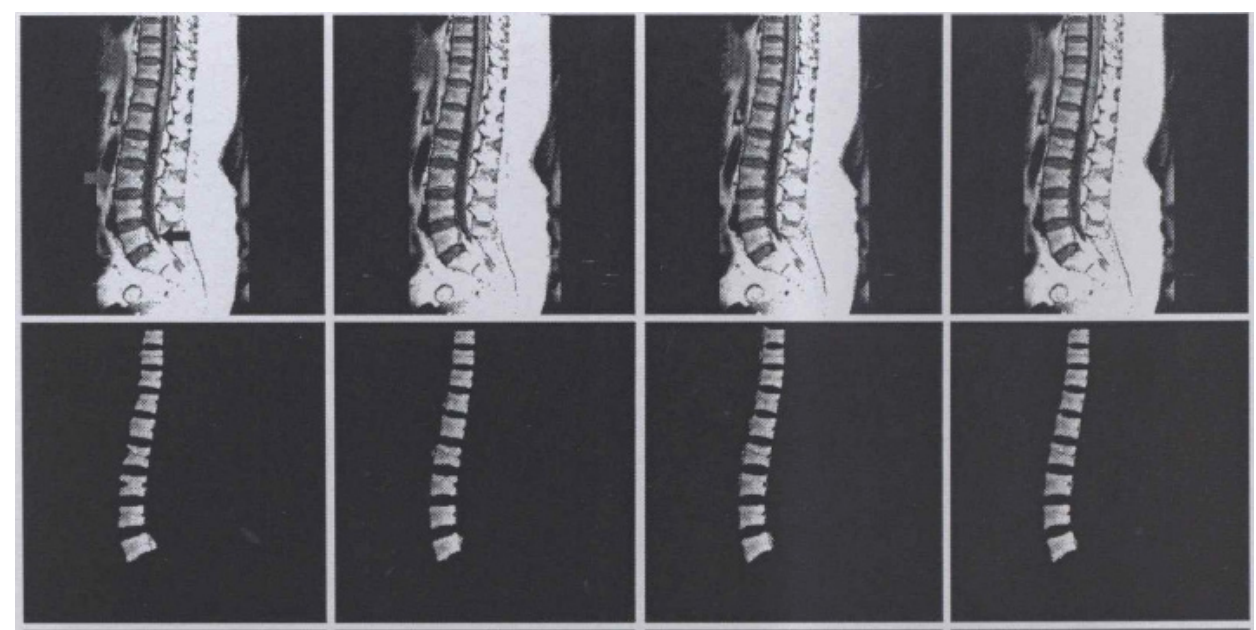

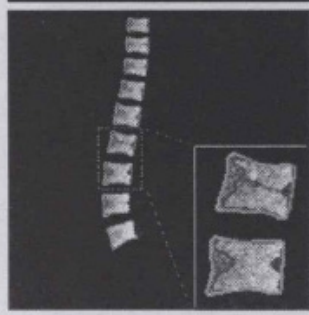

(a)

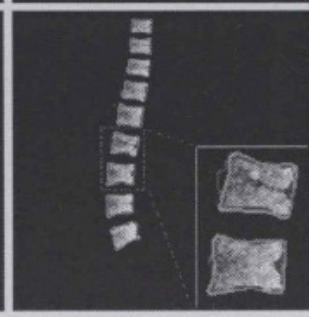

(b)

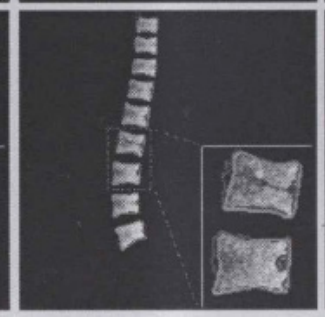

(c)

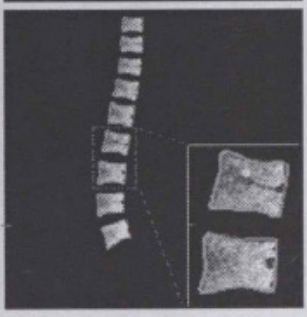

(d)

Fig. (2). The boundaries (red line) and segmentation results of the vertebral bodies. (a) NJW algorithm; (b) The Gamio algorithm; (c) The Shi algorithm; (d) The proposed algorithm;In Row3: the Gold Standard is displayed in the green line and the automatic segmentation is displayed in the red line. 
surrounding tissue and vertebral gray value is very close, but the method in this paper can accurately extract the distortion vertebral body, and the other three kinds of automatic segmentation method cannot accurately segment the vertebral body, as shown in Fig. (4). The experiment parameter settings are as follows: in NJW algorithm, $\sigma=0.05, C=65$; in Gamio algorithm, $\sigma=0.15, \quad C=40 ; \quad$ in Shi algorithm, $\sigma_{x}=\infty, \sigma_{I}=0.02, \sigma_{C}=0.15, \alpha=1, C=68$.
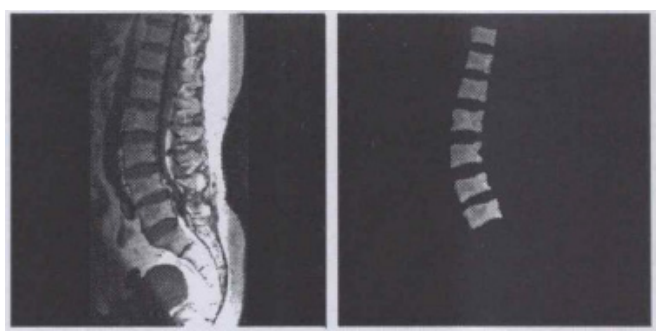

Fig. (3). Gold Standard: the boundaries (red line) and segmentation results of the vertebral bodies.

Experiment 3: T2 weighted images, patients with normal vertebral body

In spine MR images, the vertebral body of patients is not deformation, the difference of adjacent tissue surrounding vertebral body and vertebral body is larger, the segmentation of vertebral body from this kind of spine MR image is easier. The experimental results the clinical expert manual segmented and vertebral centrum brim extracted is shown in Fig. (5). As shown in Fig. (6), the NJW algorithm has the over segmentation phenomena to a certain extent (blue ar- row); this algorithm can accurately segment vertebral body, smooth and clear, overcome the over segmentation phenomenon in the NJW algorithm, the segmentation of vertebral contours and vertebral body shape is close to the manual segmentation results. In the experiment, parameter settings are as follows: in NJW algorithm, $\sigma=0.1, C=38$; in Gamio algorithm, $\sigma=0.12, C=40$; in Shi algorithm, $\sigma_{x}=\infty$, $\sigma_{I}=0.2, \sigma_{C}=0.025, \alpha=1, C=63$.

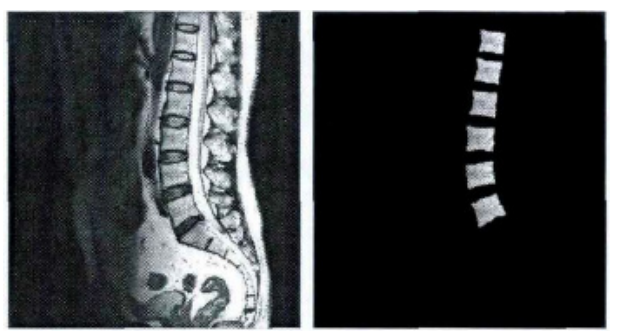

Fig. (5). Gold Standard: the boundaries (red line) and segmentation results of the vertebral bodies.

Experiment 4: Patients vertebral body with lesions, vertebral degeneration, intervertebral disc herniation (T2 weighted image)

With the increase of age, the spine will be prone to disease, such as vertebral degeneration, intervertebral disc herniation. Fig. (9) is the vertebral bodies contour a clinical expert manual segmented and the vertebral body extracted. Because the influence of imaging technology, vertebral body has bright spot, as shown in Fig. (10). The segmentation
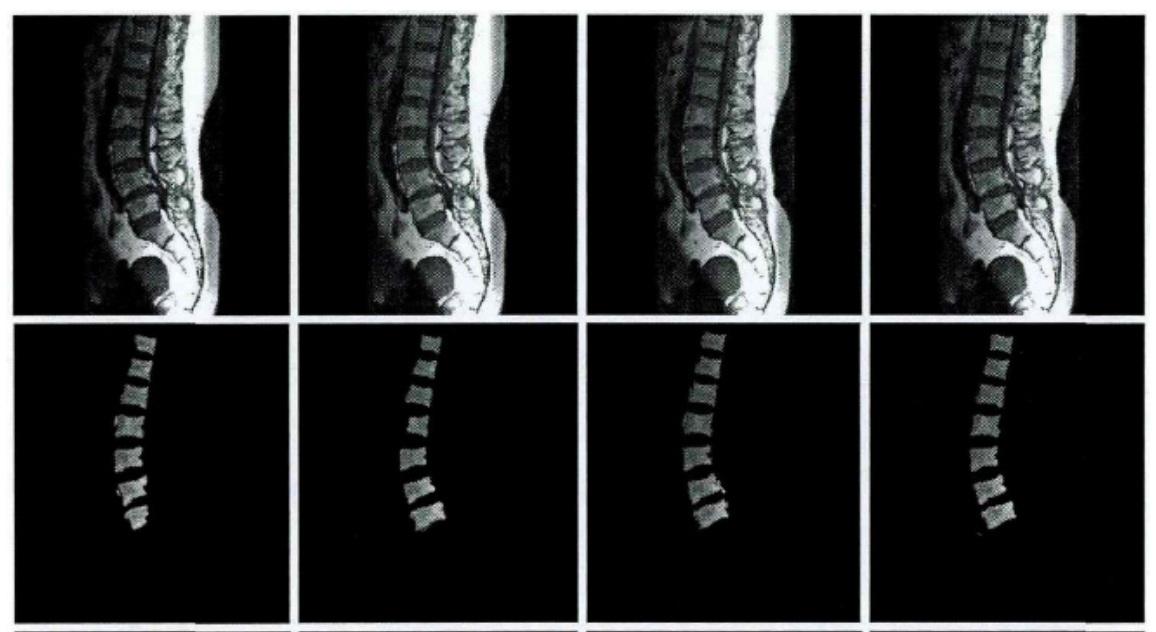

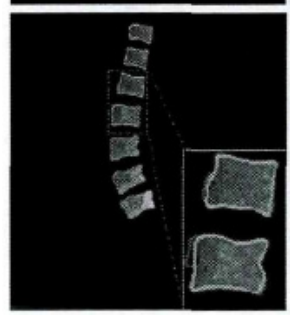

(a)

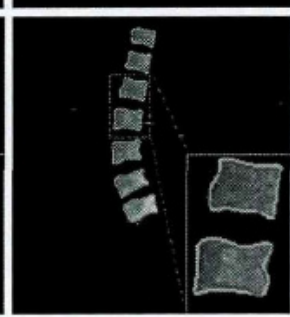

(b)

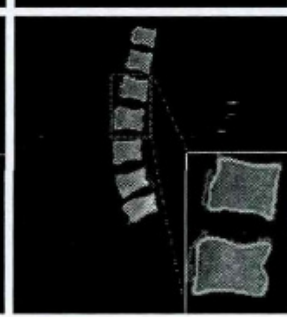

(c)

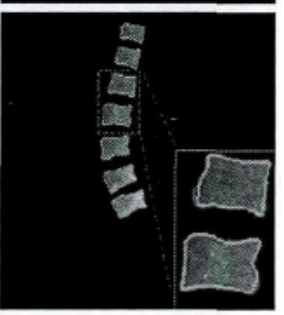

(d)

Fig. (4). The boundaries (red line) and segmentation results of the vertebral bodies. (a) NJW algorithm; (b) The Gamio algorithm; (c) The Shi algorithm; (d) The proposed algorithm; In Row3: the Gold Standard is displayed in the green line and the automatic segmentation is displayed in the red line. 


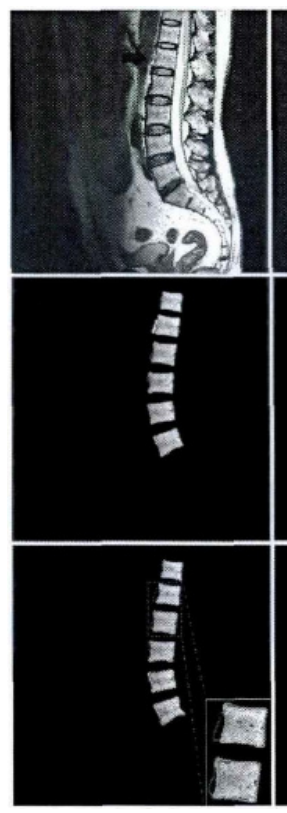

(a)

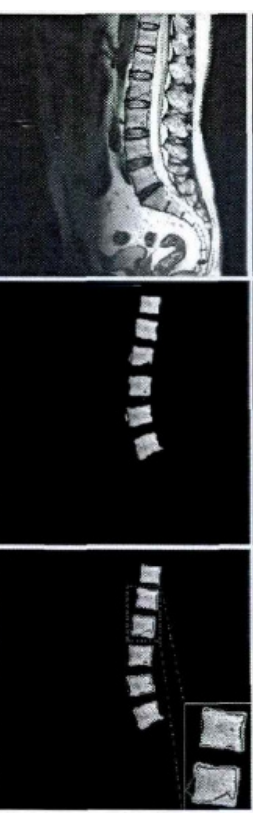

(b)

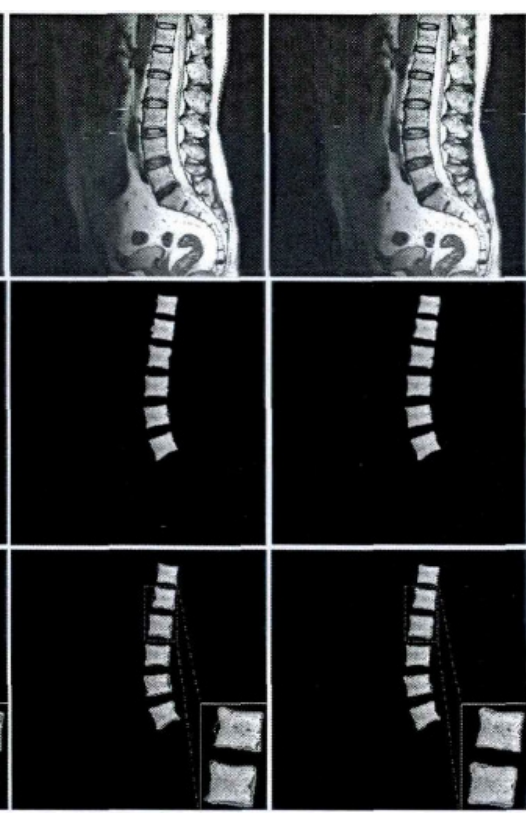

(c)

(d)

Fig. (6). The boundaries (red line) and segmentation results of the vertebral bodies, (a) NJW algorithm; (b) The Gamio algorithm; (c) The Shi algorithm; (d) The proposed algorithm; In Row3: the Gold Standard is displayed in the green line and the automatic segmentation is displayed in the red line.
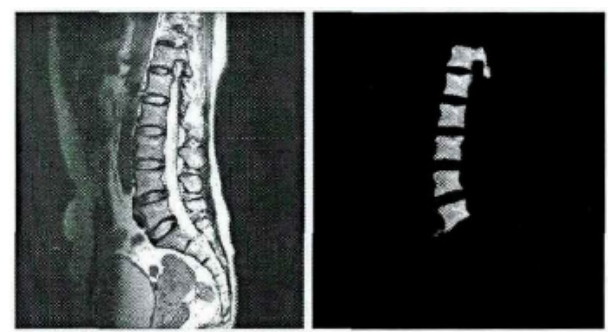

Fig. (7). Gold Standard: the boundaries (red line) and segmentation results of the vertebral bodies.

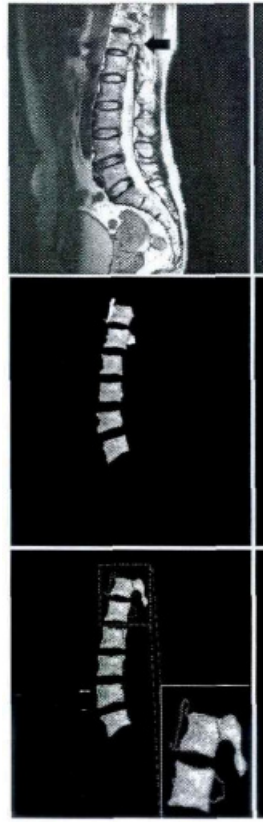

(a)

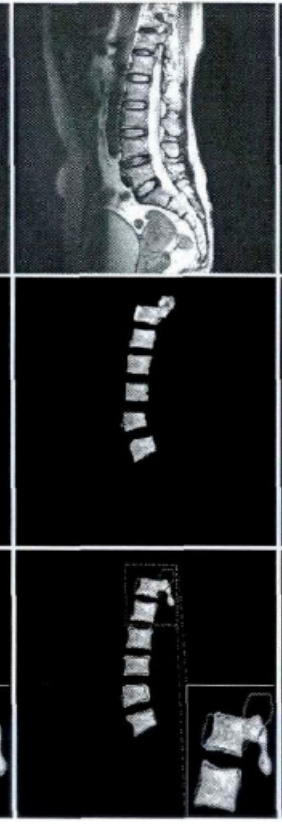

(b)

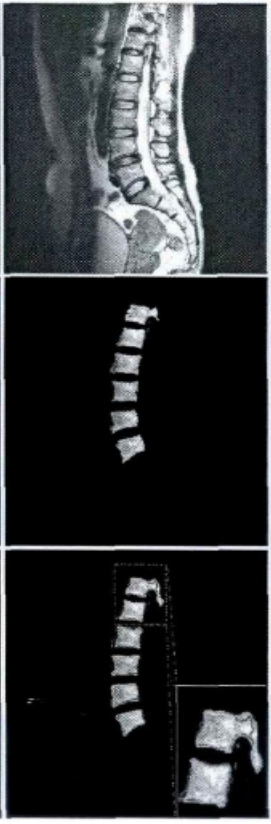

(c)

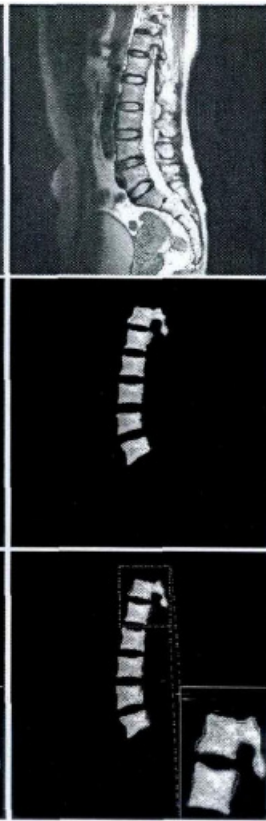

(d)

Fig. (8). The boundaries (red line) and segmentation results of the vertebral bodies. (a) NJW algorithm; (b) The Gamio algorithm; (c) The Shi algorithm; (d) The proposed algorithm; In Row3: the Gold Standard is displayed in the green line and the automatic segmentation is displayed in the red line. 


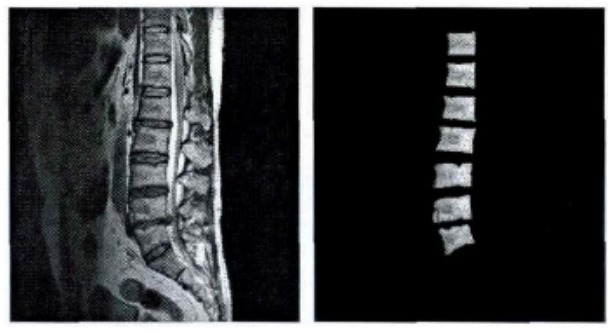

Fig. (9). Gold Standard: the boundaries (red line) and segmentation results of the vertebral bodies.

result from this kind of spine MR image is slightly difference, NJW algorithm cannot accurately segment the degenerative change vertebral body; even if the vertebral body shape is changed, the algorithm can accurately segment vertebral body, smooth and clear, vertebral contours segmented and vertebral body shape is more close to the manual segmentation results. In the experiment, parameter settings are as follows: in NJW algorithm, $\sigma=0.1, C=50$; in Gamio algorithm, $\sigma=0.1, C=40$; in Shi algorithm, $\sigma_{x}=\infty$, $\sigma_{I}=0.22, \sigma_{C}=0.05, \alpha=1, C=90$.

In order to evaluate objectively the feasibility of the proposed algorithm, Table 1 lists the comparison results of manual segmentation algorithm and automatic segmentation algorithm proposed in this paper, the feasibility of this algorithm is stick out a mile from the table. The Dice value characterizes the similarity of manual segmentation and automatic segmentation results, ME represents the probability of error segmentation, HD represents the maximum distance from the manual segmentation and edge of automatic segmentation. The bigger of the Dice value shows that the manual segmentation and automatic segmentation results consistency is better; the smaller of the ME, shows that the number of pixels error segmented is less. From Table $\mathbf{1}$ shows, the accuracy rate of this algorithm is above $96 \%$, the wrong segmentation rate is below $0.3 \%$, vertebral body edge segmented is most close to clinical experts outline, this algorithm can obtain the better segmentation results than other segmentation algorithms.

\subsection{Variable Comparison}

NJW algorithm and Gamio algorithm use gray information to segment vertebral body, therefore need set and adjust the parameters of gray information during the segmentation process. The Shi algorithm use gray and edge information to segment, increase the adjustment parameters. The algorithm in this paper use local neighborhood information to segment vertebral body, while introduce local contraction to avoid repeatedly manual adjusting the scale parameter $\sigma$. Table 2 lists the parameters needed to be adjusted and execution time in four automatic segmentation algorithms.

The parameters algorithm in this paper needed to be manually set is the least; algorithm execution time is the longest. Although our algorithm execution time is the longest, but our algorithm only needs to be performed once, while other algorithms need to repeatedly adjust parameters to get the most satisfactory segmentation effect, which is very time-consuming. The time of calculating similarity matrix respectively is: $\mathrm{NJW}=0.3366 \mathrm{~s}, \quad$ Gamio $=0.3571 \mathrm{~s}$, $\mathrm{Sh}=0.6864 \mathrm{~s}$, the algorithm of Improved $=0.7650 \mathrm{~s}$. Because

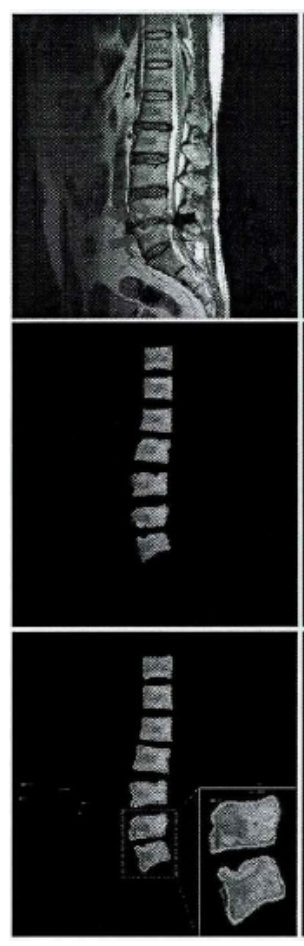

(a)

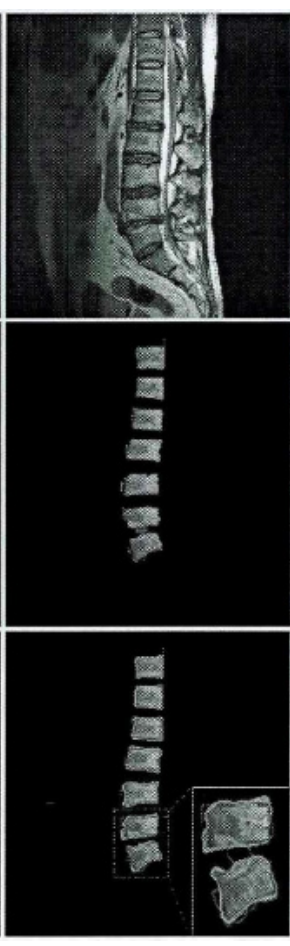

(b)

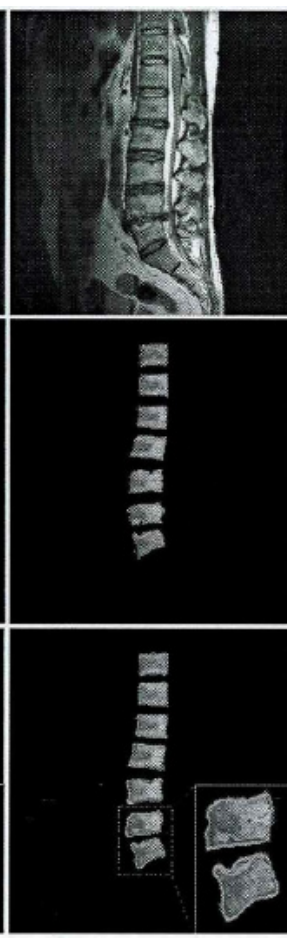

(c)

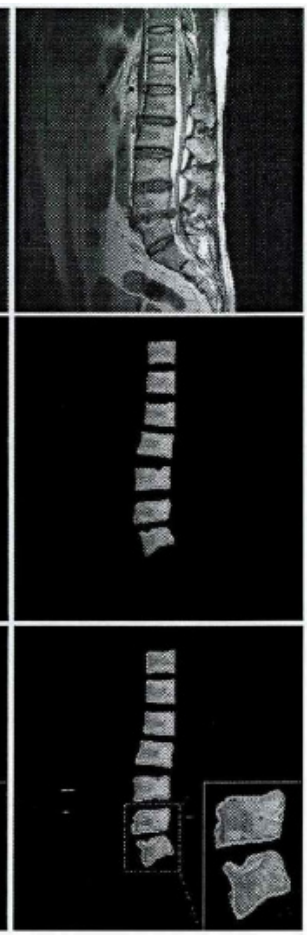

(d)

Fig. (10). The boundaries (red line) and segmentation results of the vertebral bodies, (a) NJW algorithm; (b) The Gamio algorithm; (c) The Shi algorithm; (d) The proposed algorithm; In Row3: the Gold Standard is displayed in the green line and the automatic segmentation is displayed in the red line. 
Table 1. The comparisons between the four algorithms.

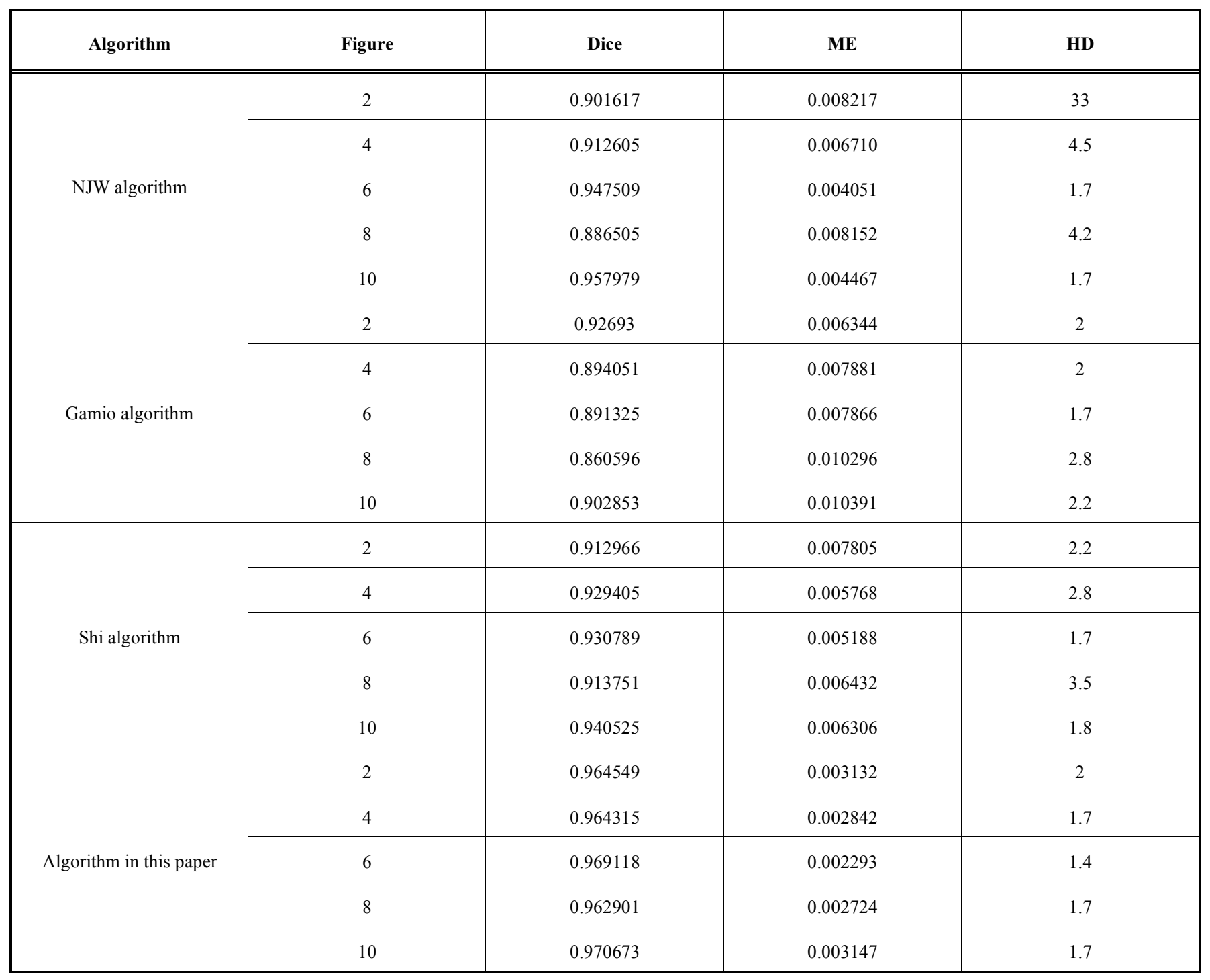

Table 2. The comparisons between the four algorithms.

\begin{tabular}{|c|c|c|c|c|}
\hline gray information & $\sqrt{ }(\sigma)$ & $\sqrt{ }(\sigma)$ & $\sqrt{ }\left(\sigma_{I}\right)$ & $x$ \\
\hline position information & $\times$ & $x$ & $\sqrt{ }\left(\sigma_{x}\right)$ & $\times$ \\
\hline Weight & $\times$ & $\times$ & $\sqrt{ }(\alpha)$ & $x$ \\
\hline clustering number & $\sqrt{ }(C)$ & $\sqrt{ }(C)$ & $\sqrt{ }(C)$ & $\sqrt{ }(C)$ \\
\hline Time/s & 51.47 & 53.29 & 15.25 & 57.01 \\
\hline
\end{tabular}

*The $\sqrt{ }$ denotes the algorithm has the parameter, if not is $\times$. 


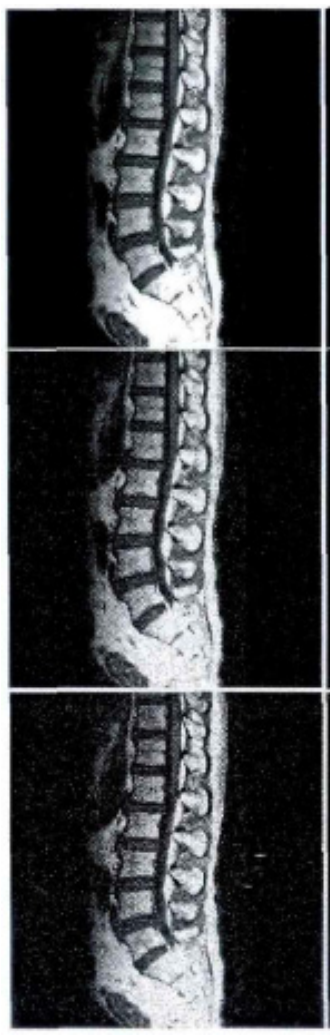

(a)

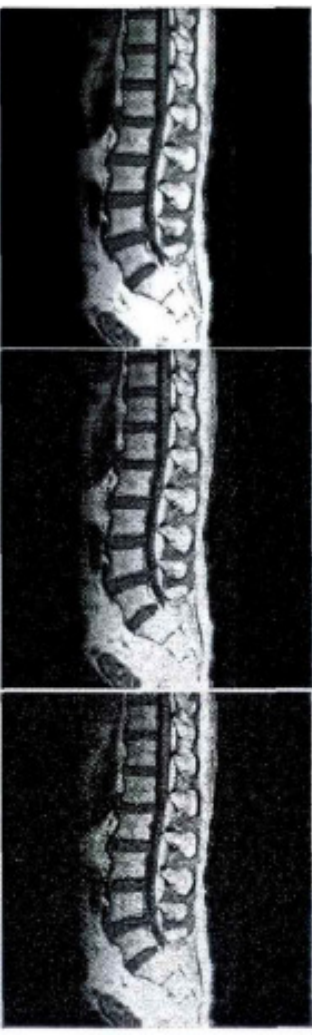

(b)

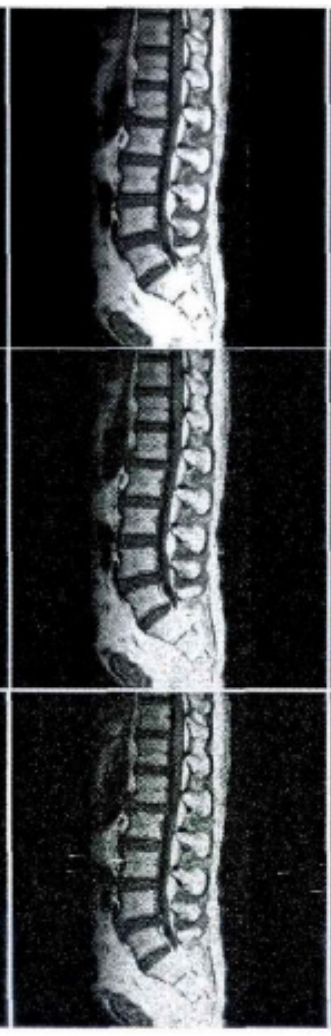

(c)

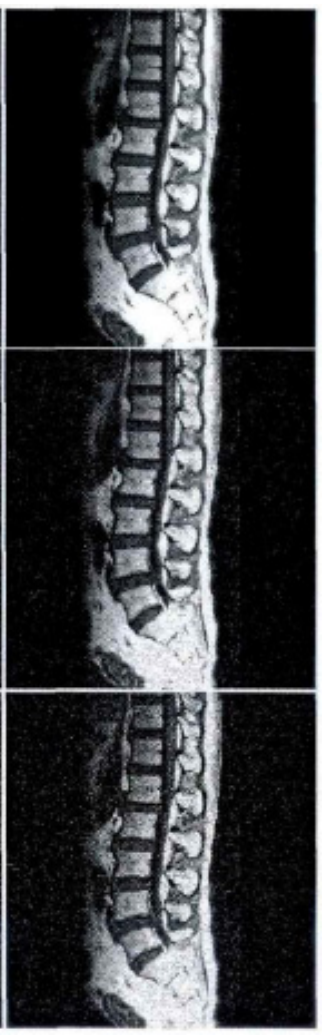

(d)

Fig. (11). Comparisons of the four algorithms on segmentation results with zero-mean Gaussian white noise. (a) The segmentation of NJW algorithm; (b) The Gamio's algorithm; (c) The Shi's algorithm; (d) The proposed algorithm.

the algorithm in this paper needs to compute the local neighborhood information, the time of calculating similarity matrix is also the most.

\subsection{Robustness Analysis}

In order to study the robustness of this algorithm against noise, we add Gauss white noise of the 0 mean different variance of on the real spine MR image, the segmentation result is shown in Figs. (11 and 12) shows Dice, ME and HD values of evaluation indexes of 4 kinds of automatic segmentation algorithm, we can see from the above results, this method has good robustness to Gauss noise.

\section{CONCLUSION}

The technology of image segmentation based on graph theory is a new segmentation technology; in the field of medical image processing is still in the research stage. NJW algorithm structure the similarity matrix, only use the feature of single pixel value, not good enough to describe an image, and need to do a lot of experiments to get the appropriate scale parameter, improve the segmentation accuracy, an image pixel distribute in different scale spaces, even at the case of obtaining a suitable scale parameter, also cannot get ideal segmentation effect. As shown in the experimental results, with the image of vertebral body and adjacent tissue gray similar and vertebral degenerative deformation the segmentation effect is not ideal, vertebral body cannot be accurately segmented, in vertebral edges appear the phenomenon of over segmentation and less segmentation.
The algorithm our proposed gives full consideration to the impact of the pixels around the pixel neighborhood, we introduce spatial structure information, based on experience the neighborhood information is combined together as the certain feature weight, that is use Gauss function weighted Z2 distance metric the similar size of two pixel nodes, so construct a new similarity matrix, improve the results of clustering and segmentation. In the choice of the scale parameter, this algorithm introduces local contraction thought, automatically assigned a scale parameter for every pixel, which makes the algorithm adaptive to image segmentation, even when an image pixel distribute in different scale space, the algorithm still has the adaptive adjusting. As shown in the experimental results, at the automatic segmentation of vertebral body from the spine MR image, with the image of vertebral body and the adjacent tissues gray similar, scoliosis, intervertebral disc deformation, displacement still has very good segmentation results and robustness. As a general segmentation method, it can be extended to other tissue segmentation.

Spine MR image segmentation is always difficult, vertebral body contour segmented using spine MR image segmentation method based on neighborhood information and Gauss weighted chi-square distance is more close to manual segmentation, and do not need to repeatedly adjust parameters, accurate segmentation result has great application value in auxiliary diagnosis for image registration, image retrieval, spinal deformity analysis, organ positioning and related diseases. 


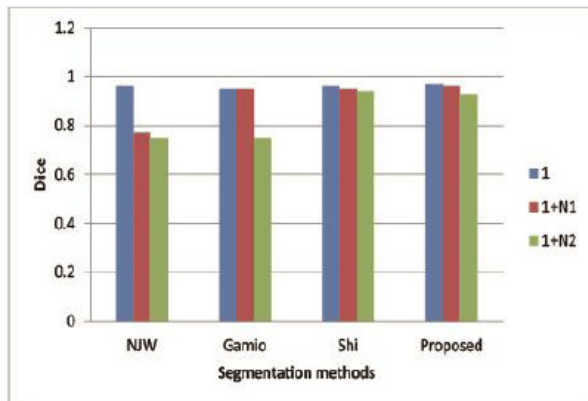

(a)

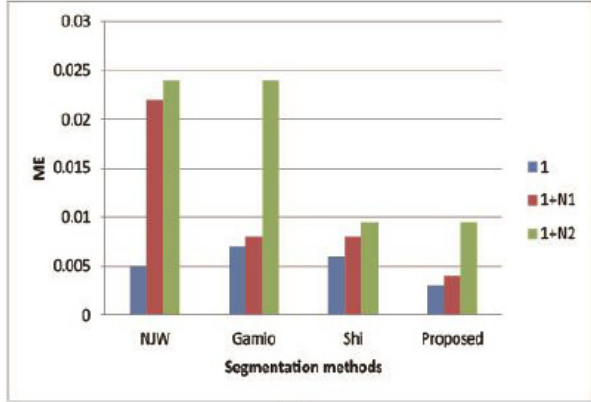

(b)

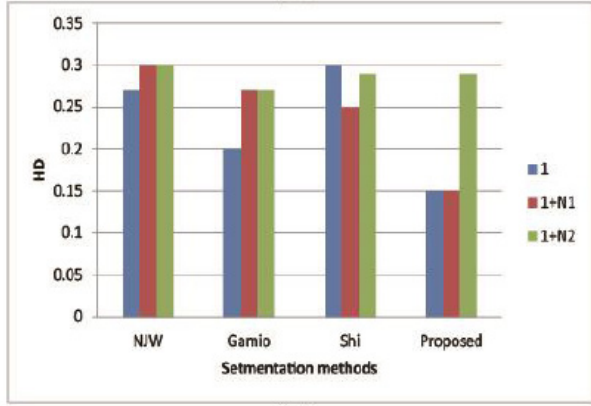

(c)

Fig. (12). Dice, ME and HD similarity measures computed for the four methods. Original image is shown in red, and the images added Gaussian white noise with variances 0.01 and 0.02 are shown in green and blue, respectively. (a) Dice similarity measure: (b) ME similarity measure; (c) HD similarity measure.

\section{CONFLICT OF INTEREST}

The authors confirm that this article content has no conflict of interest.

\section{ACKNOWLEDGEMENTS}

Declared none.

\section{REFERENCES}

[1] Liu S, Peng Y. A local region-based Chan-Vese model for image segmentation [J]. Pattern Recogn, 2012,45(7): 2769-2779.

[2] Ghugre NR, Enriquez CM, Gonzalez I, Nelson MD, Jr., Coates TD, Wood JC. MRI detects myocardial iron in the human heart [J]. Magn Reson Med, 2006, 56(3): 681-686.

[3] Kirk P, He T, Anderson LJ, et al. International reproducibility of single breathhold T2* MR for cardiac and liver iron assessment among five thalassemia centers [J]. J Magn Reson Imaging, 2010, 32(2): 315-319.

[4] Westwood MA, Firmin DN, Gildo M, et al. Intercentre reproducibility of magnetic resonance $\mathrm{T} 2 *$ measurements of myocardial iron in tbalassaemia [J], Int J Cardiovasc Imaging, 2005, 21(5): 531538.

[5] Yamamura J, Grosse R, Graessner J, Janka GE, Adam Q Fischer R. Distribution of cardiac iron measured by magnetic resonance imaging (MRI)-R*2 [J]. J Magn Reson Imaging, 2010, 32(5): 11041109.

[6] Pepe A, Positano V, Santarelli MF, et al. Multislice multiecho T2* cardiovascular magnetic resonance for detection of the heterogeneous distribution of myocardial iron overload [J]. J Magn Reson Imaging, 2006,23(5): 662-668.

[7] Saiviroonporn P, Viprakasit V, Boonyasirinant T, Khuhapinant A, Wood JC, Krittayaphong R. Comparison of the region-based and pixel-wise methods for cardiac $\mathrm{T} 2 *$ analysis in 50 transfusiondependent Thai thalassemia patients [J]. J Comput Assist Tomo, 2011,35(3): 375-381.

[8] Carpenter JP, He T, Kirk P, et al. On T2* magnetic resonance and cardiac iron [J]. Circulation, 2011,123(14): 1519-1528.

[9] Crum WR, Camara 0, Hill DL. Generalized overlap measures for evaluation and validation in medical image analysis [J]. IEEE Trans Med Imaging, 2006, 25(11): 1451-1461.

[10] Maska M, Danek 0, Garasa S, Rouzaut A, Munoz-Barrutia A, Ortiz-de-Solorzano C. Segmentation and shape tracking of whole fluorescent cells based on the Chan-Vese model [J]. IEEE Trans Med Imaging, 2013, 32(6): 995-1006.

[11] Ng AY, Jordan MI, Weiss Y. On Spectral Clustering 1 Analysis and an algorithm [J]. Proceedings of Advances in Neural Information Processing Systems Cambridge, MA: MIT Press, 2001,14: 849-856.

[12] Carballido-Gamio J, Belongie SJ, Majumdar S. Normalized cuts in 3-D for spinal MRI segmentation [J]. IEEE Trans Med Imaging, 2004,23(1): 36-44.

[13] Cour T, Benezit F, Shi J. Special segmentation with multiscale graph decomposition $[\mathrm{C}]$. In proceedings of computer vision and pattern recognition, 2005,2:1124-1131.

[14] Zelnik-Manor L, Perona P. Self-tuning spectral clustering [C]. In proceedings of NIPS, 2004, 17:16.

Received: June 10, 2015

Revised: July 29, 2015

Accepted: August 15, 2015

(C) Dan et al.; Licensee Bentham Open.

This is an open access article licensed under the terms of the (https://creativecommons.org/licenses/by/4.0/legalcode), which permits unrestricted, noncommercial use, distribution and reproduction in any medium, provided the work is properly cited. 